\title{
The Prognostic Significance of the Preoperative Platelet-Lymphocyte Ratio in Japanese Patients with Localized Breast Cancer
}

\author{
Hideya Takeuchi1 ${ }^{*}$, Seiichi Fukuyama1, Nobuhide Kubo' ${ }^{1}$, Shoji Hiroshige ${ }^{1}$, \\ Hirofumi Kawanaka1,2, Yasuji Yoshikawa ${ }^{3}$, Tokujiro Yano ${ }^{1}$ \\ ${ }^{1}$ Department of Surgery, National Hospital Organization Beppu Medical Center, Beppu, Japan \\ ${ }^{2}$ Clinical Research Institute, National Hospital Organization Beppu Medical Center, Beppu, Japan \\ ${ }^{3}$ Department of Pathology, National Hospital Organization Beppu Medical Center, Beppu, Japan \\ Email: "t3996@beppu.hosp.go.jp
}

Received 5 February 2016; accepted 2 April 2016; published 8 April 2016

Copyright @ 2016 by authors and Scientific Research Publishing Inc.

This work is licensed under the Creative Commons Attribution International License (CC BY).

http://creativecommons.org/licenses/by/4.0/

(c) (i) Open Access

\begin{abstract}
Inflammation has been shown to be a factor in tumorigenesis. The circulating platelet to lymphocyte ratio (PLR) is a representative index of systemic inflammation. In this study, we analyzed the association between preoperative PLR levels and clinicopathological variables in two hundred sixty-four Japanese patients with localized breast carcinoma. We also evaluated the prognostic significance of preoperative PLR levels using the Cox proportional hazard model. Seventy-five patients (28.4\%) had elevated PLR values, whereas 189 (71.6\%) had depressed PLR values. The PLR correlated significantly with venous invasion $(P<0.05)$. Disease-free survival rates were significantly worse among patients with elevated PLR values than among those with lower PLR value (5-year survival, $81.6 \%$ vs. $90.7 \%$, respectively; $P<0.05$ ). In multivariate analysis, elevated PLR, nuclear grade, and lymph node involvement were significant prognostic factors for disease-free survival in patients with breast carcinoma $(P<0.05)$. Preoperative PLR levels may be an independent prognostic factor in patients with breast carcinoma.
\end{abstract}

\section{Keywords}

Platelet-Lymphocyte Ratio, Breast Cancer, Inflammation

\footnotetext{
"Corresponding author.
} 


\section{Introduction}

Breast cancer is the most frequently diagnosed malignancy and one of the leading causes of cancer-related mortality in women world-wide, including Japan [1] [2]. Several factors, such as age, tumor size, histological grade, and nodal involvement, have been identified as unfavorable clinicopathological prognostic factors in breast cancer patients [3]. Additionally, molecular diagnostic tests that predict prognosis, such as Oncotype Dx, Mammaprint, and the Amsterdam 70 gene signature, may be used to obtain additional prognostic and/or predictive information. However, the high cost, lack of standardization, and regional availability of these gene expression-based tests limit their application in routine clinical practice [4]; moreover, the Japanese National Health Insurance does not support the routine evaluation for breast cancer using these kits. Therefore, routine peripheral blood-based parameters that can be obtained with fewer drawbacks would potentially be of benefit for improving patients' individualized risk assessment.

Tumor progression and metastasis arise from a cascade of steps that promote the interaction between the tumor and the host-derived stromal microenvironment. Inflammation can enhance tumor growth, invasion, and eventually metastasis [5]. In breast cancer patients, elevated inflammatory markers that are usually measured by peripheral blood-derived inflammation-based parameters, such as serum amyloid A and interleukin (IL)-6, have been shown to be associated with reduced survival [6] [7].

Platelets play an important and multifaceted role in cancer progression. For example, an elevated pretreatment platelet count has been associated with poor survival in colorectal cancer patients [8]. Lymphocytes also play a major role in cancer immune-surveillance, which suppresses tumor maturation [9]. An elevated lymphocyte count has been associated with significantly prolonged overall survival in patients with myeloma and colorectal cancer [10] [11]. Accumulating evidence suggests that a high PLR might constitute an independent adverse prognostic factor in esophageal [12], ovarian [13], and colorectal cancers [14]. However, data regarding the prognostic significance of the PLR in breast cancer are sparse [15].

The purpose of our study was to evaluate the effect of the preoperative PLR on disease-free survival of localized breast cancer patients in order to determine its prognostic significance.

\section{Patients and Methods}

A total of 399 patients with histologically confirmed breast cancer who underwent surgery at the Beppu Medical Center between February 2007 and December 2014 were included in this retrospective study. Sixty-nine of the 399 patients were excluded from our analysis, because of distant metastases at initial presentation $(n=8)$, carcinoma in situ $(\mathrm{n}=41)$, bilateral breast carcinoma $(\mathrm{n}=15)$, and male breast carcinoma $(\mathrm{n}=5)$. Among the remaining 330 patients, those with comorbidities that affected serum PLR levels, including infection $(\mathrm{n}=1)$, collagen disease $(n=1)$, and liver cirrhosis $(n=7)$, were excluded to avoid potential bias. Those with lacking PLR data $(n=38)$ or their entire set of pathological data $(n=18)$ were excluded. Finally, 264 localized breast cancer patients with a median age of 62.6 (range: 28 - 90) years at the time of diagnosis were eligible for the inclusion in this study. The patient selection criteria are summarized in Figure 1. The Ethical Committee for Clinical Research of Beppu Medical Center approved this retrospective study (2015-4).

Adjuvant therapy was administered according to the St Gallen recommendations [16]. Adjuvant chemotherapy was administered to 32 patients (12.1\%), adjuvant hormonal treatment to 129 (48.9\%), and adjuvant chemotherapy and hormonal therapy together to 97 (36.7\%). Among these patients, 24 (9.1\%) were treated preoperatively.

Follow-up care was performed at regular intervals (3-month intervals for years 1 - 5; and 6-month intervals for years 5 - 10 after diagnosis). Follow-up investigations included clinical examinations, laboratory data analyses including carcinoembryonic antigen and carbohydrate-antigen 15-3, and radio-logical assessment (computed tomography and mammography) every 12 months in years $1-5$, and years $5-10$, respectively.

\subsection{Pathological Characteristics}

Pathological data were reviewed to determine the tumor size, nuclear grade, lymph node status, hormone receptor status and human epidermal growth factor receptor 2 (HER2) status. Estrogen receptor (ER) and progesterone receptor (PgR) statuses were evaluated via immunohistochemistry (IHC). Tumors with nuclear expression $>0$ were recorded as positive. HER2 status was assessed via IHC or fluorescence in situ hybridization and was considered 


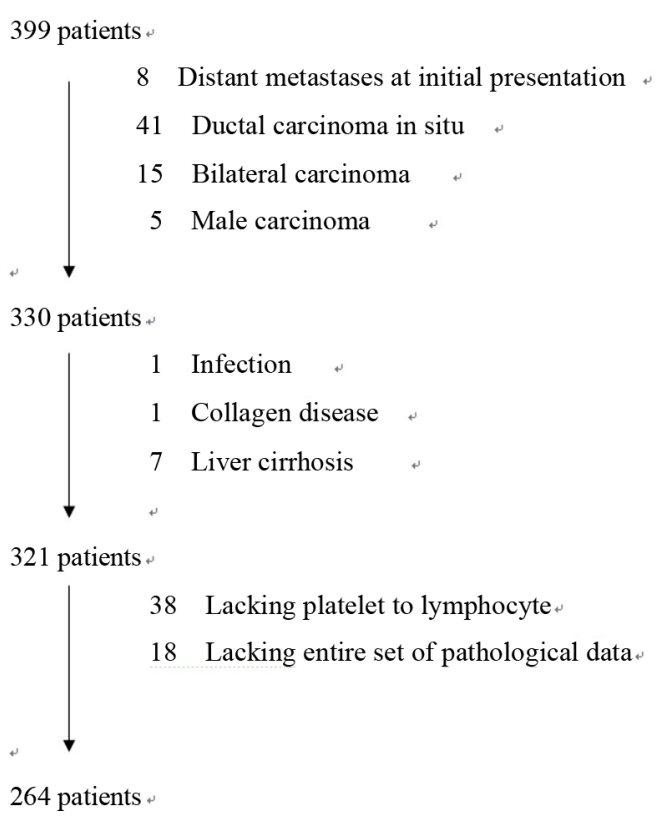

Figure 1. Flowchart illustrating the enrollment and exclusion criteria of patients with breast cancer in this study.

positive upon obtaining either an IHC score of 3 or at least a 2.2-fold stronger HER2 signal relative to the CEP 17 signal in the tumor cells [17]. Lymphatic invasion and venous invasion were defined by the identification of intravascular tumor clots in the lumina of lymphatic or blood vessels, respectively.

\subsection{PLR Measurement}

Blood samples were obtained via peripheral venous puncture before the initiation of any treatment modality. Platelets and lymphocytes were measured routinely with an automatic nephelometer (XE-5000; Sysmex Corporation, Tokyo, Japan) according to the manufacturer's instructions. Assays were performed by medical technicians who were blinded to the study subjects’ clinical information.

The PLR was calculated as the absolute platelet count divided by the absolute lymphocyte count. The ideal cutoff value for PLR was determined by receiver operating curve (ROC) analysis (Figure 2). According to the ROC, we determined an optimal cut off value of 162.3 for the PLR.

\subsection{Statistical Analysis}

The primary end point of the study was disease-free survival defined as the interval between the date of surgery and the first observation of disease relapse. Comparisons between two groups were performed using the chisquare test.

The Kaplan-Meier method was used to calculate disease-free survival rates. Significant differences in diseasefree survival were determined using log-rank tests. All variables were assessed in a Cox proportional hazard model to identify any independent variables associated with disease-free survival. Hazard ratios (HRs) estimated using the Cox analysis was reported as relative risks with corresponding 95\% confidence intervals (CIs). All statistical analyses were performed using EZR (Saitama Medical Center, Jichi Medical University, Saitama, Japan), a graphical user interface for $\mathrm{R}$ (The R Foundation for statistical Computing, Vienna, Austria). EZR is a modified version of $\mathrm{R}$ Commander designed to add the statistical functions frequently used in biostatistics [18]. A Pvalue $<0.05$ was considered significant.

\section{Results}

The mean platelet count was $22.8 \pm 5.8 \times 10^{4}$, the mean lymphocyte count was $1795 \pm 1015$, and the mean PLR 


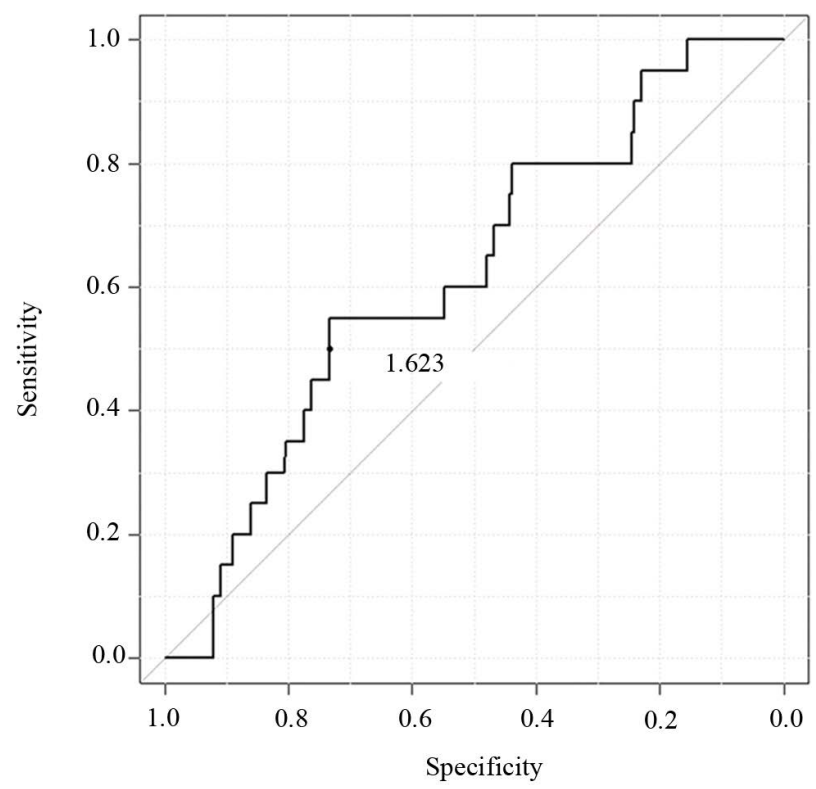

Figure 2. Receiver operating curve (ROC) for disease-free survival in patients with breast carcinoma. The ROC is represented with an area under the curve of 0.62 (95\% Confidence Interval: 0.499 0.741 ), with a sensitivity of 0.5 and a specificity of 0.734 .

was 143.6 (range; 17.4 - 454.3). Seventy five patients (28.4\%) had PLR values at or above the cutoff ( $\geq 162.3)$, and 189 patients $(71.6 \%)$ had PLR-low values $(<162.3)$.

The associations between preoperative PLR and clinicopathological variables are summarized in Table 1. Although elevated PLR had a correlative trend with ER status and lymph node involvement $(\mathrm{P}=0.055$ and 0.054, respectively), there were no significant differences between the two groups. A high PLR significantly correlated with venous invasion $(\mathrm{P}<0.05)$.

The mean follow-up period was 33 months. The disease-free survival rate was significantly lower in the PLRhigh group than in the PLR-low group (5-year survival, 81.6\% vs. 90.7\%, respectively; P < 0.05) (Figure 3). Univariate analysis revealed a significant impact of nuclear grade, ER, lymph node involvement and tumor size on disease-free survival. With respect to PLR, only a trend toward decreased survival was detected $(P=0.052)$. On multivariate analysis, an elevated PLR level, nuclear grade and lymph node involvement were independently correlated with poor prognosis for disease-free survival, with respective HRs of 3.5970 (95\% CI, 1.324 - 9.774), 3.1 (95\% CI; 1.106 - 8.691) and 3.039 (95\% CI; 1.65-8.672), respectively (Table 2).

\section{Discussion}

In the present study, we demonstrated that an elevated preoperative PLR value was significantly associated with venous invasion, and can be a significant prognostic indicator in Japanese patients with localized breast cancer on multivariate analysis. Interestingly, the HR for elevated PLR was superior to those for well-known risk factors (e.g. nuclear grade [1 - 2 vs. 3] and lymph node involvement [negative vs. positive]). This finding is consistent with previous reports for several other types of cancers [12]-[14].

Systemic inflammation is associated with the release of several pro-inflammatory mediators, such as IL-1, IL-3, and IL-6, which are known to stimulate megakaryocyte proliferation, leading to the production of blood platelets. Experimental data have shown a direct correlation between the number of circulating platelets and the level of serum VEGF, a major factor in tumor-induced angiogenesis [19], and metalloproteinase-9 secretion [20]. Furthermore, platelets negatively modulate the innate antitumor immune response through cell-induced platelet aggregation, which shields tumor cells from the major histocompatibility complex so as to escape immune surveillance by $\mathrm{T}$ cells [21]. These facts could explain that an elevated peripheral platelet count was associated with higher mortality in patients with several cancers [8] [22].

Systemic inflammation also leads to the release of a number of immunologic mediators, most notably IL-10 


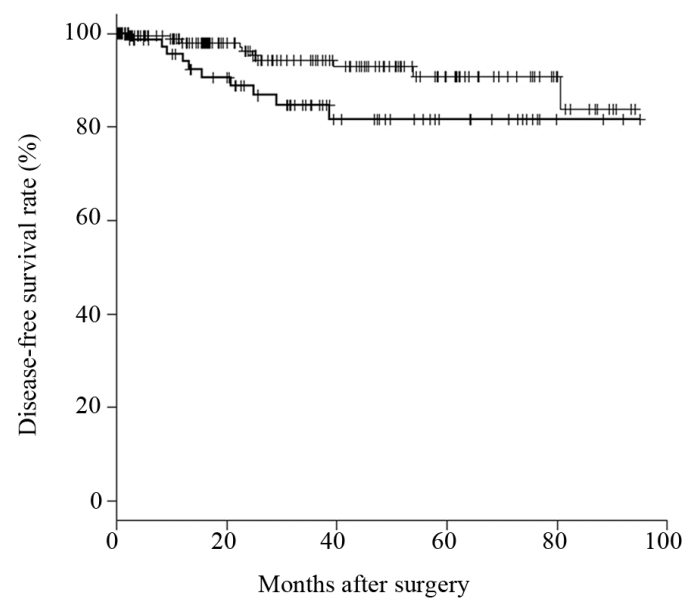

Figure 3. Kaplan-Meier analysis of disease-free survival stratified by the preoperative platelet to lymphocyte ratio (PLR) in patients with breast carcinoma. Patients with an elevated PLR value (bold line) had a significantly poorer disease-free survival rate than those with a normal PLR value (thin line) $(\mathrm{P}<0.05)$.

Table 1. Relationship between preoperative platelet to lymphocyte ratio and clinicopathological variables of patients with breast carcinoma.

\begin{tabular}{|c|c|c|c|}
\hline & No. (\%) & No. (\%) & $P$ value \\
\hline Variables & PLR < $162.3(n=189)$ & PLR $\geq 162.3(n=75)$ & \\
\hline \multicolumn{4}{|l|}{ Age } \\
\hline$<50$ years & 35 (19\%) & $18(24 \%)$ & 0.313 \\
\hline$>50$ years & 154 (81\%) & 57 (76\%) & \\
\hline \multicolumn{4}{|c|}{ Nuclear grade } \\
\hline $1-2$ & $126(67 \%)$ & 53 (71\%) & 0.846 \\
\hline 3 & 63 (33\%) & 22 (29\%) & \\
\hline \multicolumn{4}{|c|}{ Lymphatic invasion } \\
\hline Negative & 98 (52\%) & 36 (48\%) & 0.588 \\
\hline Positive & 91 (48\%) & 39 (52\%) & \\
\hline \multicolumn{4}{|c|}{ Venous invasion } \\
\hline Negative & $148(78 \%)$ & 47 (63\%) & 0.0126 \\
\hline Positive & 41 (22\%) & $28(37 \%)$ & \\
\hline \multicolumn{4}{|c|}{ Estrogen receptor } \\
\hline Negative & 33 (17\%) & $6(8 \%)$ & 0.0555 \\
\hline Positive & $156(83 \%)$ & $69(92 \%)$ & \\
\hline \multicolumn{4}{|c|}{ Progesterone receptor } \\
\hline Negative & 47 (25\%) & 22 (29\%) & 0.535 \\
\hline Positive & $142(75 \%)$ & $53(71 \%)$ & \\
\hline \multicolumn{4}{|l|}{ HER2 } \\
\hline Negative & $160(85 \%)$ & $58(77 \%)$ & 0.207 \\
\hline Positive & 29 (15\%) & 17 (23\%) & \\
\hline \multicolumn{4}{|c|}{ Lymph node involvement } \\
\hline Negative & 139 (72\%) & $46(62 \%)$ & 0.054 \\
\hline Positive & 50 (28\%) & 29 (38\%) & \\
\hline \multicolumn{4}{|l|}{ Tumor size } \\
\hline$<20 \mathrm{~mm}$ & $94(50 \%)$ & $31(41 \%)$ & 0.223 \\
\hline$>20 \mathrm{~mm}$ & 95 (50\%) & 44 (59\%) & \\
\hline
\end{tabular}

Abbreviations: HER2: human epidermal growth factor receptor 2; No.: number. 
Table 2. Univariate and multivariate analysis of clinicopathological variables that predict disease-free survival in patients with breast carcinoma using Cox proportional hazard model.

\begin{tabular}{|c|c|c|c|c|c|}
\hline \multirow[b]{2}{*}{ Variables } & \multirow[b]{2}{*}{ No (\%) } & \multicolumn{2}{|c|}{ Univariate analysis } & \multicolumn{2}{|c|}{ Multivariate analysis } \\
\hline & & $\begin{array}{c}\text { HR } \\
(95 \% \mathrm{CI})\end{array}$ & P value & $\begin{array}{c}\text { HR } \\
(95 \% \mathrm{CI})\end{array}$ & $\mathrm{P}$ value \\
\hline \multicolumn{6}{|l|}{ Age } \\
\hline$<50$ years & $53(20 \%)$ & 1 (Referent) & & 1 (Referent) & \\
\hline$>50$ years & $211(80 \%)$ & $\begin{array}{c}0.70 \\
(0.25-1.92)\end{array}$ & 0.49 & $\begin{array}{c}0.51 \\
(0.17-1.59)\end{array}$ & 0.25 \\
\hline \multicolumn{6}{|c|}{ Nuclear grade } \\
\hline $1-2$ & 179 (68\%) & 1 (Referent) & & 1 (Referent) & \\
\hline 3 & 85 (32\%) & $\begin{array}{c}2.93 \\
(1.21-7.09)\end{array}$ & 0.017 & $\begin{array}{c}3.10 \\
(1.11-8.69)\end{array}$ & 0.031 \\
\hline \multicolumn{6}{|c|}{ Lymphatic invasion } \\
\hline Negative & 134 (51\%) & 1 (Referent) & & 1 (Referent) & \\
\hline Positive & 130 (49\%) & $\begin{array}{c}2.43 \\
(0.93-6.34)\end{array}$ & 0.069 & $\begin{array}{c}1.27 \\
(0.36-4.56)\end{array}$ & 0.71 \\
\hline \multicolumn{6}{|c|}{ Venous invasion } \\
\hline Negative & 195 (74\%) & 1 (Referent) & & 1 (Referent) & \\
\hline Positive & $69(26 \%)$ & $\begin{array}{c}1.39 \\
(0.57-3.41)\end{array}$ & 0.47 & $\begin{array}{c}0.55 \\
(0.17-1.74)\end{array}$ & 0.31 \\
\hline \multicolumn{6}{|c|}{ Estrogen receptor } \\
\hline Negative & 39 (15\%) & 1 (Referent) & & 1 (Referent) & \\
\hline Positive & 225 (85\%) & $\begin{array}{c}0.32 \\
(0.12-0.84)\end{array}$ & 0.021 & $\begin{array}{c}0.30 \\
(0.06-1.50)\end{array}$ & 0.14 \\
\hline \multicolumn{6}{|c|}{ Progesterone receptor } \\
\hline Negative & $69(26 \%)$ & 1 (Referent) & & 1 (Referent) & \\
\hline Positive & 195 (74\%) & $\begin{array}{c}0.41 \\
(0.17-1.10)\end{array}$ & 0.054 & $\begin{array}{c}0.72 \\
(0.18-2.97)\end{array}$ & 0.65 \\
\hline \multicolumn{6}{|l|}{ HER2 } \\
\hline Negative & 218 (83\%) & 1 (Referent) & & 1 (Referent) & \\
\hline Positive & $46(17 \%)$ & $\begin{array}{c}1.48 \\
(0.49-4.45)\end{array}$ & 0.49 & $\begin{array}{c}0.71 \\
(0.22-2.28)\end{array}$ & 0.56 \\
\hline \multicolumn{6}{|c|}{ Lymph node involvement } \\
\hline Negative & 185 (70\%) & 1 (Referent) & & 1 (Referent) & \\
\hline Positive & 79 (30\%) & $\begin{array}{c}3.32 \\
(1.36-8.13)\end{array}$ & 0.0086 & $\begin{array}{c}3.04 \\
(1.07-8.67)\end{array}$ & 0.038 \\
\hline \multicolumn{6}{|l|}{ Tumor size } \\
\hline$<20 \mathrm{~mm}$ & 125 (47\%) & 1 (Referent) & & 1 (Referent) & \\
\hline$>20 \mathrm{~mm}$ & 139 (53\%) & $\begin{array}{c}4.35 \\
(1.44-13.14)\end{array}$ & 0.0091 & $\begin{array}{c}3.01 \\
(0.91-10.6)\end{array}$ & 0.070 \\
\hline \multicolumn{6}{|l|}{ PLR } \\
\hline$<162.3$ & 189 (72\%) & 1 (Referent) & & 1 (Referent) & \\
\hline$>162.3$ & 75 (28\%) & $\begin{array}{c}2.39 \\
(0.99-5.75)\end{array}$ & 0.052 & $\begin{array}{c}3.60 \\
(1.32-9.78)\end{array}$ & 0.012 \\
\hline
\end{tabular}

Abbreviations: CI: confidence interval; HER2: human epidermal growth factor receptor 2; HR: hazard ratio; No: number; PLR: platelet to lymphocyte ratio. 
and transforming growth factor (TGF)-B, which can result in a significant immunosuppressive effect that impairs lymphocytes' function and reduces their counts [23]. Lymphocytes have a major role in cancer immunesurveillance, which inhibits tumor cell proliferation and metastasis [24]. An increased concentration of intratumoral CD8+ cytotoxic lymphocytes in breast cancer has been strongly associated with decreased recurrence, and higher survival outcome [25]. Therefore, it is feasible that PLR calculated by these parameters could be a novel indicator of malignant potential of human tumors, and plausible that understanding the imbalances represented by the PLR may provide information regarding underlying tumor progression and prognosis in individuals with breast cancer.

Azab et al. [15] have already showed pretreatment PLR was not superior to absolute lymphocyte count alone in predicting long-term mortality in patients with breast cancer. However, their study analyzed overall survival but not disease-free survival, which can be influenced by numerous other factors including non-cancer-related death. Our results demonstrated the prognostic effect of preoperative PLR on disease-free survival in patients with breast cancer. Furthermore, the discrepancy between our results and those of Azab et al. may be attributable to the nature of the study population; Azab et al. included patients with ductal carcinoma in situ (Stage 0) and distant metastasis (Stage IV); these stages were excluded from our analysis. The prognosis of patients with Stage 0 is favorable enough that prognostic indicators are not required, whereas the prognosis of patients with Stage IV disease is unfavorable, and the decision regarding the method of treatment is based on various factors, such as physical condition and patient's consent, as well as prognostic indicators. Our results can therefore be considered more reliable and relevant than those of Azab et al. for evaluating the prognostic value of PLR in patients with localized breast cancer.

The limitations of this study include the relatively short follow-up period, the relatively small sample size, and the single-center design. The retrospective design of our study also renders it susceptible to data selection and analytical biases. Furthermore, we were unable to exclude the possibility that unequal distributions of unidentified clinicopathological parameters, such as malnutrition, severe stress, and intense physical exercise that interrelated with PLR, may also have introduced biased to our results. Even considering these limitations, however, our data indicate that an increased preoperative PLR may represent an independent prognostic factor in patients with localized breast cancer.

In summary, the results of this study suggest that an elevated preoperative PLR is likely to be associated with unfavorable tumor characteristics and significantly reduces disease-free survival time in patients with breast carcinoma. Regularly used blood-based parameters, such as PLR, are relatively easy to assess without additional hassles, making them attractive for improved individualized risk assessment. However, our data are still preliminary and should be interpreted cautiously pending validation by additional studies.

\section{Competing Interests}

The authors declare that they have no competing interests.

\section{References}

[1] Hortobagyi, G.N., de la Garza Salazar, J., Pritchard, K., Amadori, D., Haidinger, R., Hudis, C.A., Khaled, H., Liu, M.C., Martin, M., Namer, M., O’Shaughnessy, J.A., Shen, Z.Z. and Albain, K.S. (2005) The Global Breast Cancer Buden: Variation in Epidemiology and Survival. Clinical Breast Cancer, 6, 391-401. http://dx.doi.org/10.3816/CBC.2005.n.043

[2] Qiu, D.M., Katanoda, K., Marugame, T. and Sobue, T. (2009) A Joinpoint Regression Analysis of Long-Term Trends in Cancer Mortality in Japan (1958-2004). International Journal of Cancer, 124, 443-448. http://dx.doi.org/10.1002/ijc.23911

[3] Kraeima, J., Siesling, S., Vliegen, I.M., Klaase, J.M. and IJzerma, M.J. (2013) Individual Risk Profiling for Breast Cancer Recurrence: Towards Tailored Follow-Up Schemes. British Journal of Cancer, 109, 866-871. http://dx.doi.org/10.1038/bjc.2013.401

[4] Drukker, C.A., Bueno-de-Mesquita, J.M., Retel, V.P., van Harten, W.H., van Tinteren, H., Wesseling, J., Roumen, R.M., Knauer, M., van’t Veer, L.J., Sonke, G.S., Rutgers, E.J., van de Vijver, M.J. and Linn, S.C. (2013) A Prospective Evaluation of a Breast Cancer Prognosis Signature in the Observational RASTER Study. International Journal of Cancer, 133, 929-936. http://dx.doi.org/10.1002/ijc.28082

[5] Balkwill, F. and Mantovani, A. (2001) Inflammation and Cancer: Back to Virchow? Lancet, 357, 539-545. http://dx.doi.org/10.1016/S0140-6736(00)04046-0 
[6] Bachelot, T., Ray-Coquard, I., Menetrier-Caux, C., Rastkha, M., Duc, A. and Blay, J.Y. (2003) Prognostic Value of Serum Levels of Interleukin 6 and of Serum and Plasma Levels of Vascular Endothelial Growth Factor in HormoneRefractory Metastatic Breast Cancer Patients. British Journal of Cancer, 88, 1721-1726. http://dx.doi.org/10.1038/sj.bjc.6600956

[7] Pierce, B.L., Ballard-Barbash, R., Bernstein, L., Baumqartner, R.N., Neuhouser, M.L., Wener, M.H., Baumqartner, K.B., Gilliland, F.D., Sorensen, B.E., McTiernan, A. and Urich, C.M. (2009) Elevated Biomarkers of Inflammation Are Associated with Reduced Survival among Breast Cancer Patients. Journal of Clinical Oncology, 27, 3437-3444. http://dx.doi.org/10.1200/JCO.2008.18.9068

[8] Lin, M.S., Huang, J.X., Zhu, J. and Shen, H.Z. (2012) Elevation of Platelet Count in Patients with Colorectal Cancer Predicts Tendency to Metastases and Poor Prognosis. Hepatogastroenterology, 59, 1687-1690. http://dx.doi.org/10.5754/hge12277

[9] Dunn, G.P., Old, L.J. and Schreiber, R.D. (2004) The Immunobiology of Cancer Immunosurveillance and Immunoediting. Immunity, 21, 137-148. http://dx.doi.org/10.1016/j.immuni.2004.07.017

[10] Leitch, E.F., Chakrabarti, M., Crozier, J.E., McKee, R.F., Anderson, J.H., Horgan, P.G. and McMillan, D.C. (2007) Comparison of the Prognostic Value of Selected Markers of the systemic Inflammatory Response in Patients with Colorectal Cancer. British Journal of Cancer, 97, 1266-1270. http://dx.doi.org/10.1038/sj.bjc.6604027

[11] Ege, H., Gertz, M.A., Markovic, S.N., Lacy, M.Q., Dispenzieri, A., Hayman, S.R., Kumar, S.K. and Porrata, L.F. (2008) Prediction of Survival Using Absolute Lymphocyte Count for Newly Diagnosed Patients with Multiple Myeloma: a Retrospective Study. British Journal of Haematology, 141, 792-798. http://dx.doi.org/10.1111/j.1365-2141.2008.07123.x

[12] Sato, H., Tsubosa, Y. and Kawano, T. (2012) Correlation between the Pretherapeutic Neutrophil to Lymphocyte Ratio and the Pathologic Response to Neoajuvant Chemotherapy in Patients with Advanced Esophageal Cancer. World Journal of Surgery, 36, 617-622. http://dx.doi.org/10.1007/s00268-011-1411-1

[13] Asher, V., Lee, J., Innamaa, A. and Bali, A. (2011) Preoperative Platelet Lymphocyte Ratio as an Independent Prognostic Marker in Ovarian Cancer. Clinical and Translational Oncology, 13, 499-503. http://dx.doi.org/10.1007/s12094-011-0687-9

[14] Kwon, H.C., Kim, S.H., Oh, S.Y., Lee, S., Lee, J.H., Choi, H.J., Park, K.J., Roh, M.S., Kim, S.G., Kim, H.J. and Lee, J.H. (2012) Clinical Significance of Preoperative Neutrophil-Lymphocyte versus Platelet-Lymhpcyte Ratio in Patients with Operable Colorectal Cancer. Biomarkers, 17, 216-222. http://dx.doi.org/10.3109/1354750X.2012.656705

[15] Azab, B., Shah, N., Radbel, J., Tan, P., Bhatt, V., Vonfrolio, S., Habshy, A., Picon, A. and Bloom, S. (2013) Pretreatment Neutrophil/Lymphocyte Ratio Is Superior to Platelet/Lymphocyte Ratio as a Predictor of Long-Term Mortality in Breast Cancer Patients. Medical Oncology, 30, 432. http://dx.doi.org/10.1007/s12032-012-0432-4

[16] Goldhirsch, A., Wood, W.C., Coates, A.S., Gelber, R.D., Thurlimann, B., Senn, H.J. and Panel Members (2011) Strategies for Subtype-Dealing with the Diversity of Breast Cancer: Highlight of the St. Gallen International Expert Consensus on the Primary Therapy of Early Breast Cancer 2011. Annals of Oncology, 22, 1736-1747. http://dx.doi.org/10.1093/annonc/mdr304

[17] Noh, H., Eomm, M. and Han, A. (2013) Usefulness of Pretreatment Neutrophil to Lymphocyte Ratio in Predicting Disease-Specific Survival in Breast Cancer Patients. Journal of Breast Cancer, 16, 55-59. http://dx.doi.org/10.4048/jbc.2013.16.1.55

[18] Kanda, Y. (2013) Investigation of the Freely Available Easy-to-Use Software "EZR” for Medical Statistics. Bone Marrow Transplantation, 48, 452-458. http://dx.doi.org/10.1038/bmt.2012.244

[19] Bambace, N.M. and Holmes, C.E. (2011) The Platelet Contribution to Cancer Progression. Journal of Thrombosis and Haemostasis, 9, 237-249. http://dx.doi.org/10.1111/j.1538-7836.2010.04131.x

[20] Suzuki, K., Aiura, K., Ueda, M. and Kitajima, M. (2004) The Influence of Platelets on the Promotion of Invasion by Tumor Cells and Inhibition by Antiplatelet Agents. Pancreas, 29, 132-140.

[21] Benoy, I., Salgado, R., Colpaert, C., Weytjens, R., Vermeulen, P.B. and Dirix, L.Y. (2002) Serum Interleukin 6, Plasma VEGF, Serum VEGF, and VEGF Platelet Load in Breast Cancer Patients. Clinical Breast Cancer, 2, 311-315. http://dx.doi.org/10.3816/CBC.2002.n.008

[22] Brown, K.M., Domin, C., Aranha, G.V., Yong, S. and Shoup, M. (2005) Increased Preoperative Platelet Count Is Associated with Decreased Survival after Resection for Adenocarcinoma of the Pancreas. The American Journal of Surgery, 189, 278-282. http://dx.doi.org/10.1016/j.amjsurg.2004.11.014

[23] Placke, T., Kopp, H.G. and Salih, H.R. (2012) The Wolf in Sheep’s Clothing: Platelet-Derived "Pseudo Self” Impairs Cancer Cell “Missing Self” Recognition by NK Cells. Oncoimmunology, 1, 557-559. http://dx.doi.org/10.4161/onci.19367 
[24] Ownby, H.E., Roi, L.D., Isenberg, R.R. and Brennan, M.J. (1983) Peripheral Lymphocyte and Eosinophil Counts as Indicator of Prognosis in Primary Breast Cancer. Cancer, 52, 126-130. http://dx.doi.org/10.1002/1097-0142(19830701)52:1<126::AID-CNCR2820520123>3.0.CO;2-Y

[25] Mahmoud, S.M., Paish, E.C., Powe, D.G., Macmillan, R.D., Grainge, M.J., Lee, A.H., Ellis, I.O. and Green, A.R. (2011) Tumor-Infiltrating CD8+ Lymphocytes Predict Clinical Outcome in Breast Cancer. Journal of Clinical Oncology, 29, 1949-1955. http://dx.doi.org/10.1200/JCO.2010.30.5037 